\title{
Reciprocal regulation of IL-6 and IL-10 balance by HGF via recruitment of heme oxygenase- 1 in macrophages for attenuation of liver injury in a mouse model of endotoxemia
}

\author{
MIYUKI KAMIMOTO ${ }^{1}$, SHINYA MIZUNO $^{1}$ and TOSHIKAZU NAKAMURA ${ }^{1,2}$ \\ ${ }^{1}$ Division of Molecular Regenerative Medicine, Department of Biochemistry and Molecular Biology, \\ Osaka University Graduate School of Medicine, 2-2-B7 Yamadaoka, Suita, Osaka, 565-0871, Japan
}

Received November 20, 2008; Accepted January 15, 2009

DOI: 10.3892/ijmm_00000219

\begin{abstract}
Acute liver injury is a clinical hallmark of endotoxemia regarding the features of septic organ failure. In this process, interleukin (IL)-6 and IL-10 are key contributors for eliciting pro- and anti-inflammatory responses, respectively. In contrast, heme oxygenase-1 (HO-1) provides a defense mechanism against endotoxemia by controlling the IL-6/IL-10 balance, but how higher levels of HO-1 are sustained under pathological conditions remains unknown. Using a mouse model of endotoxemia, we provide evidence to show that hepatocyte growth factor (HGF) enhances HO-1 expression in macrophages, thereby up-regulating IL-10 and downregulating IL-6 productions. Lipopolysaccharide (LPS)-treated mice manifested acute liver injury similar to that observed in septic patients, while administration of recombinant HGF enhanced expression of HO-1 by hepatic macrophages in vivo. As a result, HGF blocked the onset of hepatic injuries in LPS-treated mice. More importantly, when an HO-1 inhibitor (Sn-PP) was administered with HGF into LPS-treated mice, the protective effects of HGF against hepatic injury were attenuated. Furthermore, Sn-PP partially restored the HGFmediated decrease in plasma IL-6 levels, while it inhibited the HGF-stimulated increase in plasma IL-10 levels. In the
\end{abstract}

Correspondence to: Professor Toshikazu Nakamura, ${ }^{2}$ Present address: Kringle Pharma Joint Research Division for Regenerative Drug Discovery, Center for Advanced Science and Innovation, Osaka University, 2-1 Yamadaoka, Suita, Osaka 565-0871, Japan

E-mail: nakamura@casi.osaka-u.ac.jp

Abbreviations: HGF, hepatocyte growth factor; HO-1, heme oxygenase-1; IL-6, interleukin-6; IL-10, interleukin-10; SIRS, Systemic immune response syndrome; LPS, Lipopolysaccharide; TUNEL, TdT-mediated dUTP-biotin nick end-labeling; ALT, alanine aminotransferase; FBS, fetal bovine serum; PCR, polymerase chain reaction; PAI-1, plasminogen activator inhibitor-1

Key words: systemic immune response syndrome, hypercytokinemia, acute liver injury, host defense system, growth factor therapy culture of macrophages (Raw264.7), HGF enhanced the LPSmediated HO-1 induction, and this effect was abolished by cycloheximide, but not by actinomycin-D, thus suggesting that a post-transcriptional pathway is involved in HGF-mediated up-regulation of HO-1. Based on the current data, we conclude that up-regulation of HO-1 plays an important role in HGFmediated hepatoprotection during endotoxemia, by favoring production of IL-10 over IL-6.

\section{Introduction}

Currently, the prevalence of sepsis is increasing worldwide due to an emergence of infectious disorders $(1,2)$. Multiple organ failure often occurs during pathological processes of hyper-coagulation and inflammation (1). Systemic immune response syndrome (SIRS) is the clinical expression of an abnormal, generalized inflammatory reaction in organs distant from the initiating insult (2). Acute liver failure is a clinical hallmark of endotoxemia regarding the features of SIRS, progression to multi-organ dysfunction and functional immunoparesis (2). Indeed, clinical reports documented the association of infection with encephalopathy through activation of SIRS, with attendant poor prognosis (3). During endotoxin-induced organ injury, interleukin-6 (IL-6) is critical to the onset of inflammation and hyper-coagulation (4). In contrast, IL-10 is an anti-inflammatory regulator produced by macrophages and counteracts the effects of IL-6-mediated pathological events (5). Thus, the balance between IL-6 and IL-10 is considered an important determinant of the clinical outcome of SIRSrelated liver injuries (2).

In contrast to progression of SIRS, there is a counterregulatory immune defense system to suppress the onset of SIRS. One such counter-regulatory system is heme oxygenase-1 (HO-1), produced mainly by macrophages (6). HO-1 is the rate-limiting enzyme for heme metabolism, converting heme to biliverdin and enabling both release of iron and generation of carbon monoxide (6). The HO-1-mediated release of these products has a protective effect in vivo under various pathological conditions $(6,7)$. Indeed, patients with an HO-1 gene mutation(s) show inflammatory phenotypes in several organs (8), while HO-1-deficient mice have reduced IL-10, but increased IL-6 production, and are sensitive to endotoxic challenges (9). These findings demonstrate that HO-1 
negatively regulates IL-6 production, and positively regulates IL-10 expression, thereby minimizing the onset of endotoxininduced organ injuries. Therefore, it is important to elucidate the mechanism by which HO-1 expression levels are regulated at a molecular level(s). However, physiological regulation of HO-1, IL-6 and IL-10 production levels in macrophages during endotoxemia remains to be investigated.

Hepatocyte growth factor (HGF) was originally identified and cloned as a potent mitogen for hepatocytes $(10,11)$. HGF acts on various types of cells through its receptor, c-Met, and exhibits pleiotropic activities during embryogenesis and tissue repair $(12,13)$. In the liver, HGF protects the parenchyma by stimulation of hepatocyte proliferation and inhibition of functional cell loss $(14,15)$. Indeed, endogenous and exogenous HGF prevents acute hepatic failure in rodents, associated with the anti-apoptotic and anti-necrotic effect of HGF on hepatocytes (16-18). On the other hand, plasma HGF levels markedly increase in septic patients (19), and macrophages sustain high levels of c-Met/HGF receptor in a culture model of endotoxic challenge (20). These backgrounds prompted us to examine whether HGF modulates the balance between inflammatory and anti-inflammatory molecules in macrophages during endotoxemia.

Using a mouse model of endotoxemia, we provide evidence to show that HGF targets macrophages and alters the balance of IL- 6 and IL-10 by increasing HO- 1 levels, contributing to attenuation of acute hepatic injury. It is believed that the preventive effect of HGF on acute hepatitis is due to direct anti-apoptotic and anti-necrotic actions of HGF on hepatocytes (16-18). In this study, we emphasized the role of 'macrophage events' in HGF-mediated therapeutic outcomes, especially under macrophage-initiated pathological conditions, such as SIRS.

\section{Materials and methods}

Animals. Eight-week-old female mice (C57B/6J strain) were purchased from Slc (Hamamatsu, Japan). Lipopolysaccharide (LPS, E. coli O111:B4) was obtained from Sigma (St. Louis, $\mathrm{MO})$. Organ injuries were induced via a single injection of LPS at a dose of $6 \mathrm{mg} / \mathrm{kg}$ (i.p.), since our preliminary study showed that this dose stably induces hepatic damages in mice (not shown). All mice were housed under specific pathogenfree conditions.

HGF treatment. Recombinant human HGF (rh-HGF) was purified from the culture medium of $\mathrm{CHO}$ cells that were transfected with an expression vector containing HGF cDNA $(14,16,18)$. The purity of rh-HGF was $>98 \%$, as verified using SDS-PAGE. To test the effects of HGF on endotoxic injury, mice were simultaneously treated with either rh-HGF ( $1 \mathrm{mg} / \mathrm{kg} / \mathrm{time}$, s.c.) or saline and the LPS challenge, followed by a second injection $12 \mathrm{~h}$ post-LPS-challenge. Likewise, normal mice were treated with rh-HGF to examine if HGF alone modulates induction of HO-1 in vivo. In another experiment, the HO-1 inhibitor, tin protoporphyrin IX (Sn-PP, Alexis, San Diego, CA), was injected at a dose of $30 \mu \mathrm{M} / \mathrm{kg} 2 \mathrm{~h}$ after rh-HGF treatment (Fig. 5A). Six mice were sacraficed, and the liver tissues were removed and fixed in either $70 \%$ ethanol or $10 \%$ formaldehyde for histological examination, as described below. Remaining tissues were immediately frozen in liquid nitrogen and stored at $-80^{\circ} \mathrm{C}$ for biochemical analyses.

Blood chemistry. To evaluate hepatic injury, alanine aminotransferase (ALT) levels were measured in plasma of septic mice, using a kit (GPT/GOT test Wako, Wako, Osaka, Japan), as previously reported (18). The concentrations of IL-6 and IL-10 in plasma were determined using an enzyme-linked immunosorbent assay (ELISA) kit (Endogen, Woburn, MA).

Histopathology. Liver tissues were embedded in paraffin and cut into $4-\mu \mathrm{m}$ sections that were subsequently stained with hematoxylin and eosin (HE). The liver injury score was determined using a previously described method, as follows, 0 , no injury; 1 , mild injury to some or all portal areas; 2 , moderate injury to some or all portal areas; 3 , marked injury to all portal areas (21).

Immune fluorescence. To detect HO-1 in injured tissue, antimouse HO-1 rabbit IgG antibody (SPA895, StressGen, Victoria, Canada) (1:100) was applied to tissue slices (thickness, $5 \mu \mathrm{m}$ ) at $4^{\circ} \mathrm{C}$ overnight, followed by incubation with the secondary Alexa-546-labeled anti-rabbit IgG antibody (Invitrogen, Carlsbad, CA). To confirm localization of HO- 1 in macrophages, sections were incubated with antiHO-1 IgG and anti-mouse Mac1 rat IgG (BD Pharmingen, San Diego, CA) overnight, followed by detection of fluorescence using Alexa 546- or 488-labeled secondary antibodies (Invitrogen). In this method, HO-1 and Mac1 were visualized as red and green images, respectively, under a confocal microscopy (LSM-PASCAL, Carl Zeiss, NY). According to the fluorescence image, the percentage of HO-1-positive cells was determined either by counting >200 Mac1-positive or -negative cells. The overall means of these parameters in each group were calculated based on individual values $(n=6)$. To identify HGFtargeted cells, we detected tyrosine-phosphorylated c-Met (p-Met) in 70\%-ethanol-fixed sections, as follows. Tissue sections were incubated with anti-p-Met-1234 rabbit IgG (Biosource, Camarillo, CA) and anti-mouse Mac1 rat IgG, followed by the secondary (i.e., fluorescence-conjugated) antibodies, as described above.

Immunohistochemistry. Using the anti-HO-1 rabbit IgG (SPA895) as the primary antibody, HO-1-positive signal was detected, based on a previous immunoperoxidase technique (18). The HO-1-positive cells were counted in $>15$ fields per mouse (x200). Furthermore, apoptotic cell death of hepatocytes was evaluated by a TdT-mediated dUTP-biotin nick endlabeling (TUNEL) method (18). In brief, the ratios of TUNELpositive cells to $>1,000$ hepatocytes were determined, and the findings were expressed as a hepatocyte apoptosis score. The overall means of these parameters in each group were calculated based on individual values $(n=6)$.

Cell culture. Raw264.7, a mouse macrophage cell line, was obtained from the American Type Culture Collection (Manassas, VA) and maintained in DMEM (Gibco, Palo Alto, $\mathrm{CA}$ ) including $10 \%$ fetal bovine serum (FBS) at $37^{\circ} \mathrm{C}$ in air with $5 \% \mathrm{CO}_{2}$. After washing twice with saline, LPS (100 ng/ml) was added to the cultures of Raw264.7 $\left(5 \times 10^{4}\right.$ cells $\left./ \mathrm{cm}^{2}\right)$ 

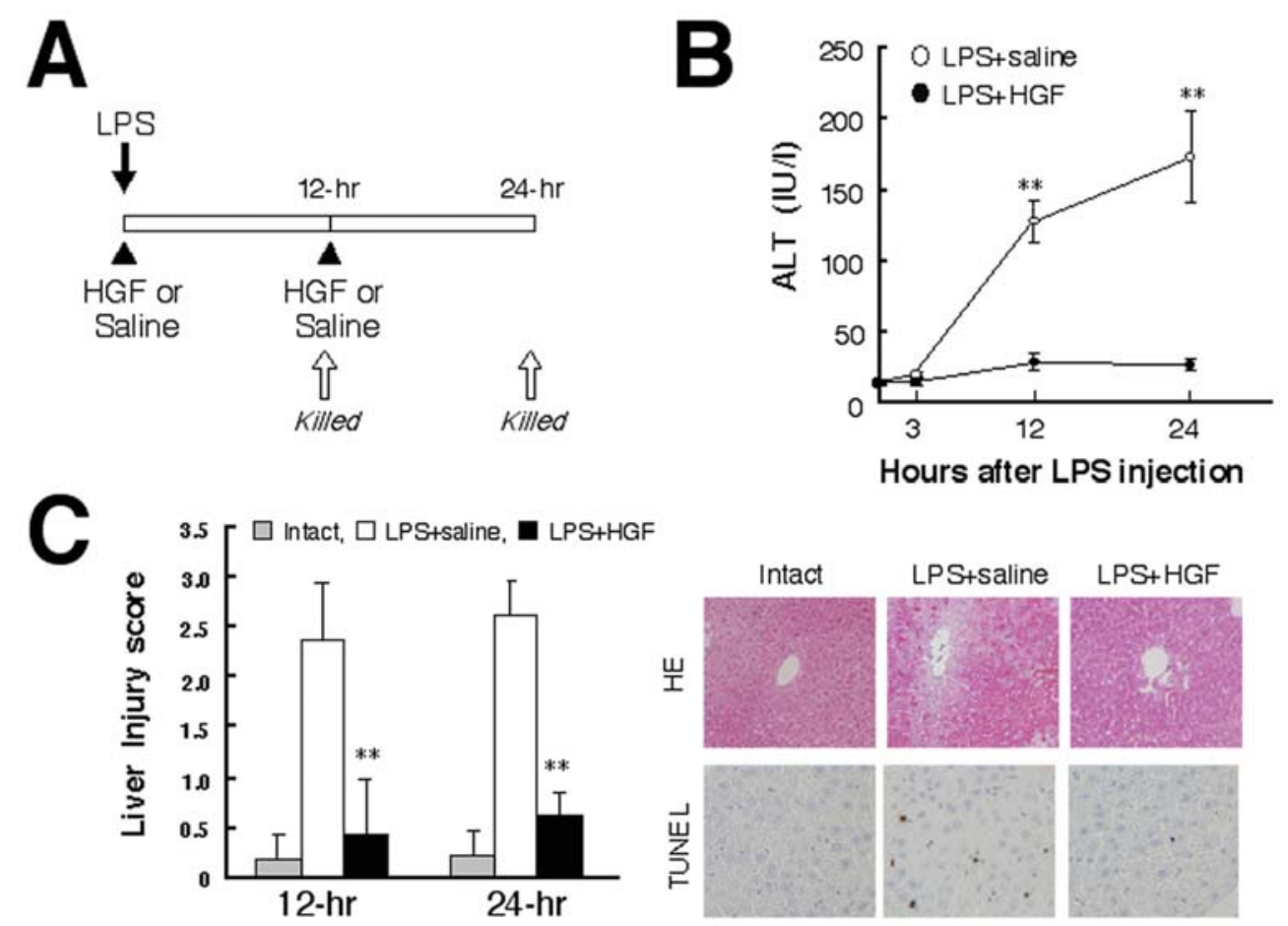

Figure 1. Protective effect of HGF against liver injury in a murine model of endotoxemia. (A) Experimental protocol for rh-HGF therapy in LPS-treated mice. (B) Changes in plasma ALT levels after LPS treatment in mice. (C) Attenuation of hepatic injuries by rh-HGF in LPS-treated mice. Left, alterations in histological injury scores $12-24 \mathrm{~h}$ post-LPS challenge. Right, typical morphological findings of degenerative changes (x280, H.E. staining) and apoptosis (x330, TUNEL staining) in the liver $24 \mathrm{~h}$ after LPS challenge. Data are expressed as mean $\pm \mathrm{SD},(\mathrm{n}=6)$. Statistical analysis, ${ }^{*} \mathrm{p}<0.05 ;{ }^{* *} \mathrm{p}<0.01 \mathrm{compared}$ with saline group at the same time points.

without FBS, up to $12 \mathrm{~h}$ post LPS challenge, because cell survival was unchanged $(>90 \%)$ within this short-term culture (not shown). The LPS-treated cells were co-incubated with rh-HGF (10, 30 and $100 \mathrm{ng} / \mathrm{ml})$ for 3, 6 and $12 \mathrm{~h}$ under FBSfree conditions. The cells were harvested at the time points indicated for Western blotting or real-time polymerase chain reaction (PCR), as described below. To explore the effects of HGF on the pathway by which HO-1 is synthesized, either cycloheximide (Nacalai, Kyoto, Japan) or actinomycin-D (Calbiochem, San Diego, CA) was added at $1 \mu \mathrm{g} / \mathrm{mg}$ to the culture 6-12 $\mathrm{h}$ after addition of LPS and rh-HGF. To determine the role of HO-1 in IL-6/IL-10 balance, Sn-PP was added to the Raw264.7 cultures based on a previously described method (22) with slight modifications. In brief, Sn-PP (30 $\mu \mathrm{M})$ was added to Raw264.7 cells, concomitantly with LPS, plus HGF $(100 \mathrm{ng} / \mathrm{ml}$ each). The culture supernatants were harvested after a 12-h incubation and subjected to ELISA, as described for the animal model.

Western blot analysis. Liver tissue homogenates or lysates of Raw264.7 cells were prepared in lysis buffer (50 mM Tris$\mathrm{HCl}, 150 \mathrm{mM} \mathrm{NaCl}, 25 \mathrm{mM}$ ß-glycerophosphate, $1 \%$ Triton $\mathrm{X}-100,10 \%$ glycerol, $5 \mathrm{mM}$ EDTA, $1 \mathrm{mM}$ phenyl methyl sulfonyl fluoride, $0.7 \mathrm{mg} / \mathrm{ml}$ pepstatin-A, $1 \mathrm{mg} / \mathrm{ml}$ leupeptin, and $1 \%$ aprotinin, $\mathrm{pH} 7.5$ ). These samples were subjected to SDS-PAGE and then transferred to PVDF membranes. Antimouse HO-1 IgG or anti-ß-actin (Sigma) was applied to PVDF membranes, followed by reaction with peroxidaselabeled secondary antibodies (Dako, Glostrup, Denmark). Positive signals were visualized using an ECL kit and semiquantified by use of an electronic densitometer (18).
Likewise, phospho-Met and total Met signals in hepatic tissues or Raw264.7 cells were detected, as reported (18).

Real-time quantitative PCR. Total RNA was prepared from the liver, using ISOGEN (Nippon Gene, Tokyo, Japan). Total RNA (1 mg) was reverse-transcribed into the first strand of cDNA with a random hexaprimer using Superscript II reverse transcriptase (Life Technologies Inc., Rockville, MD) (18). Mouse HO-1 mRNA levels were quantified using an ABI PRISM 7700 system (Perkin Elmer, Foster City), according to the manufacturer's instructions (for primer designs see catalogue no Mm00516004_m1).

Statistical analysis. A Student's t-test, ANOVA analysis, or Mann Whitney U-test was used to compare the group means, and a value of $\mathrm{p}<0.05$ was considered significant.

\section{Results}

Prevention of acute liver injury by HGF in LPS-treated mice. Using LPS-treated mice, we examined the effects of HGF on SIRS (including hyper-cytokinemia). Mice were injected with rh-HGF (1 mg/kg/12-h, s.c.), concomitantly with a single injection of LPS (6 mg/kg, i.p.) (see, Fig. 1A). As a result, rh-HGF strongly inhibited the increase in plasma ALT levels observed in the saline-treated group at 12 and $24 \mathrm{~h}$ after the LPS challenge ( $24 \mathrm{~h}, 25.2 \pm 4.3$ vs. $171.2 \pm 32.1 \mathrm{IU} / \mathrm{dl}, \mathrm{p}<0.01$ ) (Fig. 1B). Consistent with biochemical data, there was a significant difference in the hepatic injury scores between the saline and HGF groups (Fig. 1C, left). Histological examination revealed that destructive lesions, such as necrotic changes in 

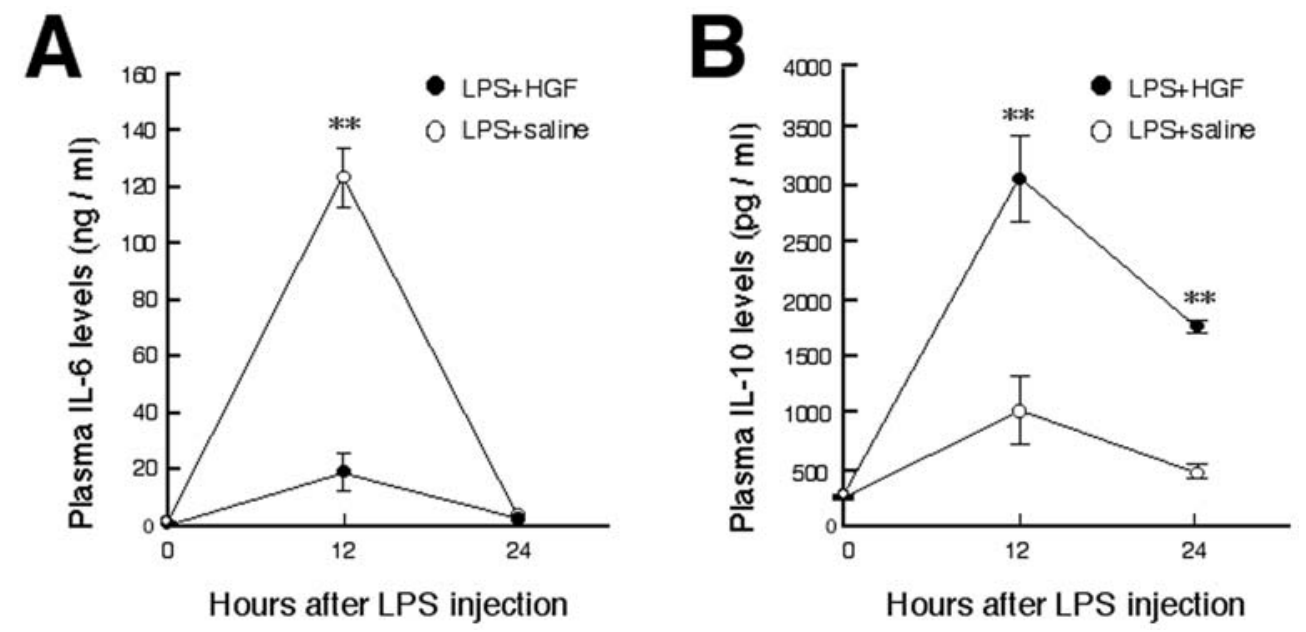

Figure 2. Effects of HGF on plasma IL-6 and IL-10 levels in mice after LPS challenge. (A) Inhibitory effect of HGF on the increase in plasma IL-6 levels in mice. (B) HGF-mediated increases in plasma IL-10 levels 12 and $24 \mathrm{~h}$ after LPS challenge. Data are expressed as mean \pm SD, (n=6). Statistical difference, ${ }^{* *} \mathrm{p}<0.01$ compared with the saline group at the same time points.

hepatocytes and clot formation in peri-portal areas, were evident $24 \mathrm{~h}$ after the LPS challenges, whereas HGF almost completely inhibited LPS-induced hepatitis in the mice (Fig. 1C, HE staining). Furthermore, rh-HGF administration strongly inhibited the apoptotic cell death of hepatocytes (Fig. 1C, TUNEL staining). Indeed, the hepatocyte apoptosis score was markedly reduced in LPS-treated mice after rh-HGF administration, as compared with that of saline-treated endotoxicemic mice ( $24 \mathrm{~h}, 1.17 \pm 1.1$ in $\mathrm{HGF}$ group vs. $4.78 \pm 1.34 \%$ in saline group, $\mathrm{p}<0.05)$. These data indicate anti-necrotic and anti-apoptotic outcomes of HGF, as reported in other models of experimental hepatitis $(16,18)$.

Changes in plasma IL-6 and IL-10 levels in LPS-treated mice after rh-HGF administration. To identify the HGF-mediated protective mechanism, we next focused on changes in plasma IL-6 and IL-10 concentrations. There was an apparent increase in plasma IL-6 concentration $12 \mathrm{~h}$ after the LPS injection. HGF inhibited the increase in plasma IL-6 to $13 \%$ of saline control concentrations $(12 \mathrm{~h}, 18.2 \pm 6.9$ vs. $122.7 \pm 10.7 \mathrm{ng} / \mathrm{ml}$, p<0.01) (Fig. 2A). In contrast, plasma IL-10 concentrations $12 \mathrm{~h}$ after the LPS injection were increased 2.2-fold, but had returned to basal levels within $24 \mathrm{~h}$ (Fig. 2B). Of note, rh-HGF treatment increased circulating levels of IL-10 3-fold compared with saline-treated LPS mice (12 h, 3039 \pm 387.3 vs. $1006 \pm 291.7 \mathrm{pg} / \mathrm{ml}, \mathrm{p}<0.01)$. Twenty-four hours after the LPS challenge, blood IL-10 levels in HGF-treated endotoxic mice remained 3-fold greater than plasma IL-10 levels in endotoxic mice (Fig. 2B). This result delineates a new role for HGF, correcting the cytokine imbalance, which is responsible for the manifestations of septic syndrome $(2,9)$.

HGF targets macrophages to increase HO-1 levels in vivo. We determined if HGF treatment activates c-Met under endotoxic stresses. In LPS-untreated control mice, rh-HGF did not elicit c-Met activation, whereas c-Met tyrosin phosphorylation was seen in LPS-treated livers $12 \mathrm{~h}$ after rh-HGF injections (Fig. 3A-I). This is consistent with previous reports that show that c-Met activation occurs in injured organs, but not in intact tissues $(23,24)$. We then focused on interstitial macrophages, since they are a major source of HO-1, IL-6 and IL-10 (4-7). In the liver of LPS-treated mice, phospho-Met signal was weak in Mac1-positive cells (including infiltrated macrophages and possibly, Kupffer cells) (Fig. 3A-II), due to possible loss of endogenous HGF activation, as reported (25) (see Discussion). In contrast, rh-HGF (i.e., active form of HGF) enhanced positive p-Met signals in Mac1-positive cells (Fig. 3A-II) (and in part, in hepatocytes), indicating that HGF directly targets macrophages under endotoxemia.

Second, we explored the effects of HGF on macrophages, focusing on HO-1 expression. Western blot analysis revealed that $\mathrm{HO}-1$ was expressed in the liver $12 \mathrm{~h}$ after LPS challenge (Fig. 3B). Hepatic HO-1 expression was not induced by HGF alone, but HGF treatment enhanced LPS-induced HO-1 expression (Fig. 3B), associated with attenuation of hepatic injury (Fig. 1B). In this process, HGF did not alter the level of HO-1 mRNA (Fig. 3C). Immunohistochemically, HO-1positive signals were detected mainly in interstitial cells, but not in hepatocytes (Fig. 3D, left), the same as septic patients (26). HGF further increased the number of the HO-1 positive cells $(24 \mathrm{~h}, 46.0 \pm 3.9$ vs. $16.3 \pm 4.1$ cells/x200 field, $\mathrm{p}<0.01)$ (Fig. 3D, right). HO-1 was identified in Mac1-positive cells (i.e., account for $70 \%$ of total $\mathrm{HO} 1$-positive cells), as shown by double fluorescent images (Fig. 3E-I). There were significant increases in the number of HO1-positive macrophages in LPS-treated mice after rh-HGF injections (Fig. 3E-II). HO-1positive signal was also seen in Mac1-negative cells, along sinusoidal walls, while HGF mildly increased HO-1 expression in these cells (Fig. 3D-II), indicating the predominant role of HGF in HO-1 expression by macrophages. In these experiments, HGF did not alter the number of macrophage per se (not shown). Therefore, we speculate that HGFenhanced HO-1 expression is due to accumulation of excess HO-1 in individual cells, rather than an increase in the number of HO-1-producing macrophages.

Possible mechanism of HGF for the enhancement of LPSinduced HO-1 expression. To test this speculation, we explored the mechanism by which HGF increases HO-1 expression levels in macrophages. In cultured Raw264.7 macrophages, 

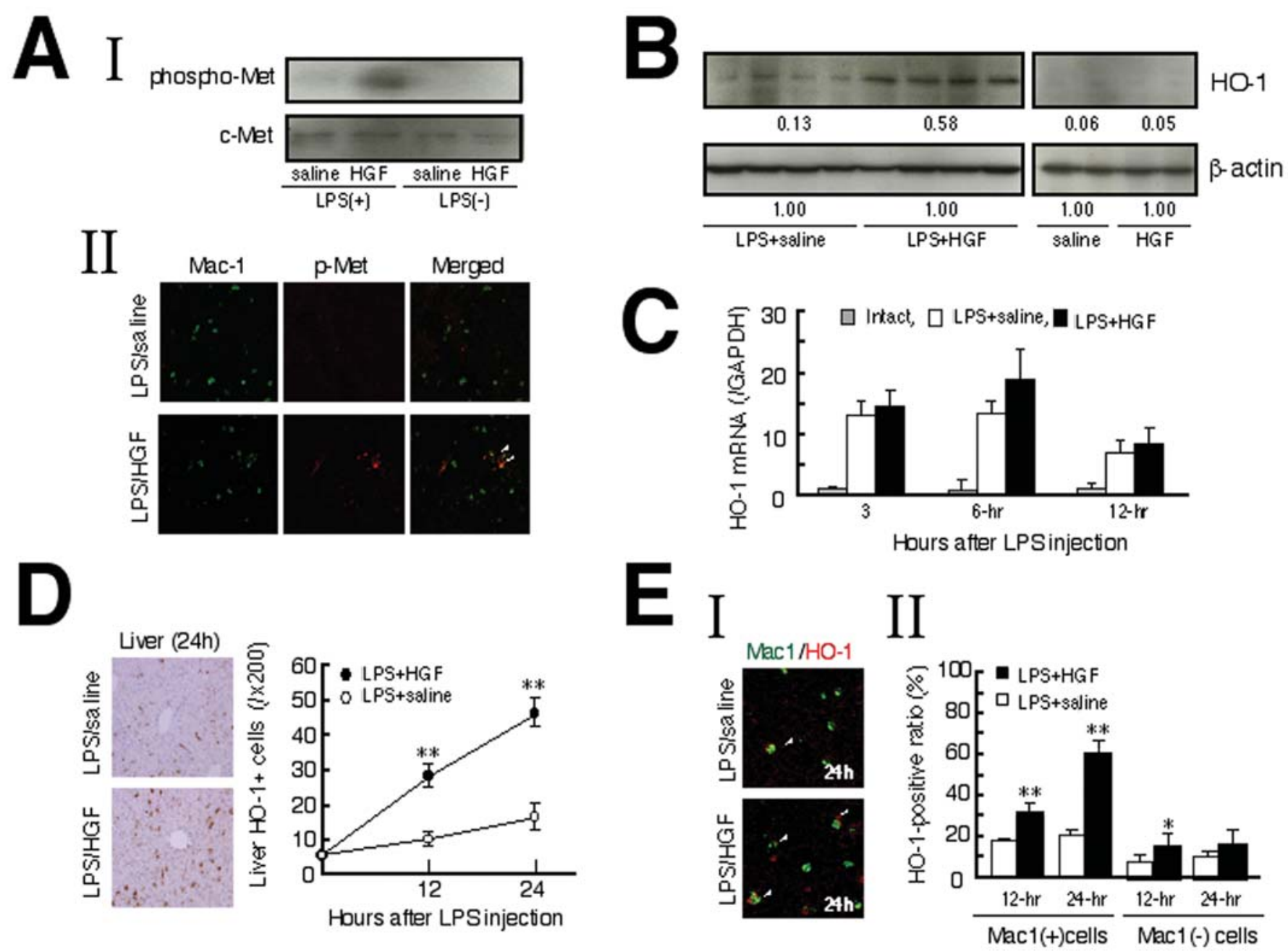

Figure 3. HGF enhances HO-1 expression in hepatic macrophages of LPS-treated mice. (A) I, Changes in c-Met tyrosine phosphorylation and total c-Met expression of hepatic tissue (12 h post-LPS-challenge), as evidenced by Western blotting; II, typical fluorescence images showing c-Met activation in macrophages. Fluorescence: Green, Mac1; Red, p-Met. Arrows indicate double-positive signals. (B) Immunoblotting showing HGF-induced increase in HO-1 expression. Liver tissues were harvested $12 \mathrm{~h}$ after LPS treatment and were subjected to biochemical analyses, as described in Methods. (C) Changes in HO-1 mRNA levels of the livers after LPS treatment, as evaluated by real-time PCR. (D) HGF-induced increase in the number of HO-1-positive cells. Livers were harvested 12-24 h after LPS challenge and were subjected to an immunoperoxidase technique (18). HO-1-positive cells were counted in >10 randomly selected fields (x200) in hepatic sections. (E). Quantitative data to show the enhancing effect of HGF on LPS-induced HO-1 expression. I, Typical images showing HO-1 expression by hepatic macrophages; II, Changes in the HO-1-positive ratio to macrophages, or to non-macrophages (\%). All data are expressed as mean $\pm \mathrm{SD},(\mathrm{n}=6)$. Statistical analysis, ${ }^{*} \mathrm{p}<0.05 ;{ }^{*} \mathrm{p}<0.01$ compared with the saline group at the same time points.

HGF elicited c-Met tyrosine phosphorylation at $15 \mathrm{~min}$ (Fig. 4A) and $2 \mathrm{~h}$ after LPS addition (not shown). Likewise, HGF alone elicited c-Met activation under LPS-free conditions, which differed from the data obtained from normal mice, due to the possibility that Raw264.7 cells undergo injurious (i.e., serum-starved) stress in vitro. We then examined the effect of HGF on HO-1 expression. Western blotting revealed that HO-1 was induced at $12 \mathrm{~h}$ in response to an LPS challenge (Fig. 4B). Consistent with the in vivo data, HGF enhanced LPS-mediated induction of HO-1, when Raw264.7 cells were incubated with $100 \mathrm{ng} / \mathrm{ml} \mathrm{HGF}$ for $12 \mathrm{~h}$ (Fig. 4B). In contrast, $\mathrm{HGF}$ alone did not alter the very low levels of HO-1 protein at $12 \mathrm{~h}$ (Fig. 4B) or at any time point after LPS addition (data not shown). Real-time PCR revealed that LPS markedly increased HO-I mRNA levels. For example, $6 \mathrm{~h}$ after LPS treatment, HO-1 mRNA levels increased 4-fold (Fig. 4C). HGF slightly enhanced LPS-mediated HO-1 transcription, but no significant difference was observed between the LPS and LPS plus HGF groups. Therefore, we hypothesize that a posttranscriptional pathway is involved in the increase of HO-1 protein levels by HGF in LPS-treated Raw264.7 cells. Cycloheximide (an inhibitor of ribosome protein synthesis) was added to cultures of Raw264.7 cells after $6 \mathrm{~h}$, because HO-1 protein levels increased dramatically between 6 and $12 \mathrm{~h}$ after addition of LPS, without changes in HO-1 mRNA (not shown). Cycloheximide diminished the HGF-mediated increase in HO-1 observed $12 \mathrm{~h}$ post-LPS addition (Fig. 4D-I). In contrast, actinomycin-D (an inhibitor of RNA transcription) did not interfere with the ability of HGF to enhance HO-1 accumulation (Fig. 4D-II), thus supporting our hypothesis.

Modulation of IL-6 and IL-10 levels by HGF in LPS-challenged Raw264.7 cells. We next determined whether the increase in HO-1 expression by HGF in LPS-stimulated macrophages leads to changes in IL-6/IL-10 balance, using the culture model of Raw264.7 cells. Just as the in vivo experiment (Fig. 2), 
A

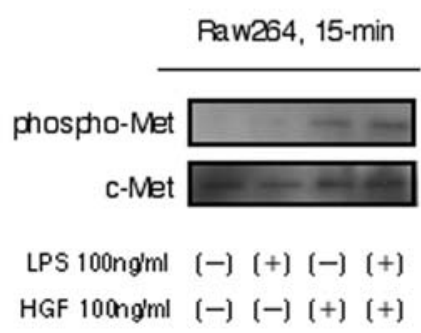

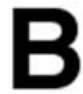

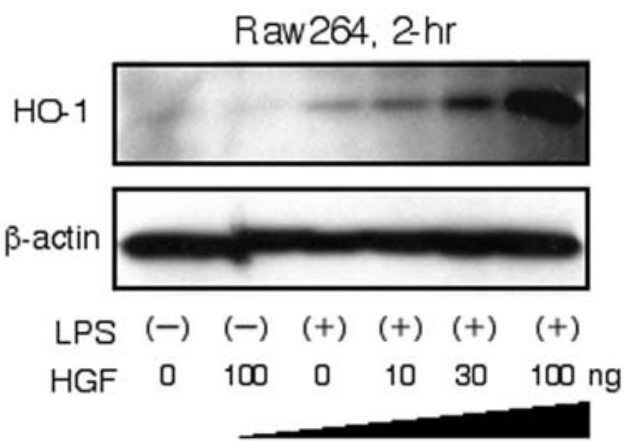

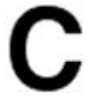

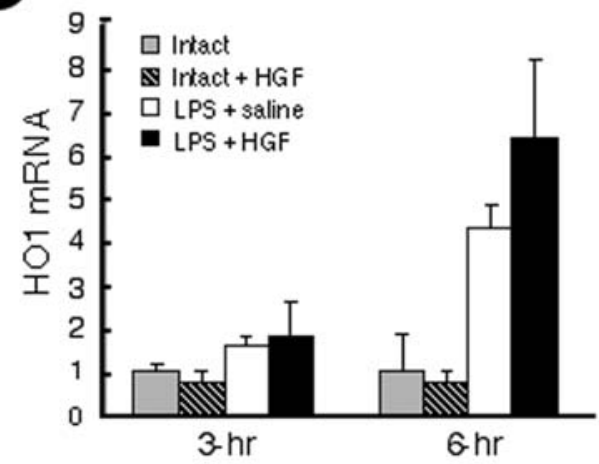

D I

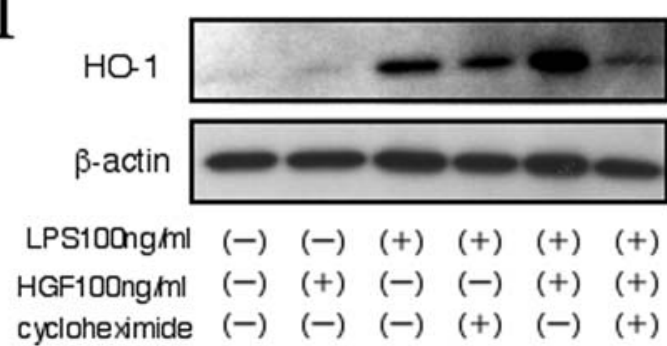

II

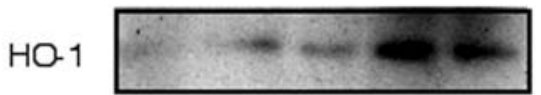

$\beta$-actin

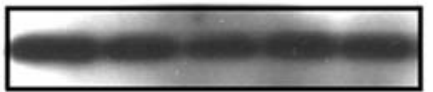

LPS100ngml (-) (+) (+) (+) (+)

HGF100ng/ml (-) (-) (-) (+) (+)

Actinomycin-D (-) (-) (+) (-) (+)

Figure 4. Possible mechanism by which HGF increases HO-1 in macrophages in vitro. (A) c-Met tyrosine phosphorylation of Raw264.7 cells after each treatment, as visualized by immunoblotting. (B) Western blot analysis showing HGF-included enhancement of HGF on LPS-induced HO-1 induction. The total lysate of Raw264.7 cells was obtained $12 \mathrm{~h}$ after the LPS challenge, and subjected to Western blot analysis, as previously described (18). (C) Real-time PCR for quantification of HO-1 mRNA levels. Total RNA was isolated 3 and $6 \mathrm{~h}$ after addition of LPS to the cultures and then was subjected to real-time PCR. (D) Possible involvement of a post-transcriptional pathway in up-regulation of HO-1 by HGF. I, Western blot showing that the HGF-mediated increase in HO-1 expression is blocked by cycloheximide (CHX). Six hours after LPS addition, CHX $(1 \mu \mathrm{g} / \mathrm{ml})$ was added to the culture, and $12 \mathrm{~h}$ after the LPS challenge, total cell lysate was obtained. II, Minimal effect of actinomycin-D (Act-D) on the HGF-mediated increase in HO-1. Act-D (1 $\mu \mathrm{g} / \mathrm{ml})$ was added to Raw264.7 cells 6-12 h post-LPS challenge.
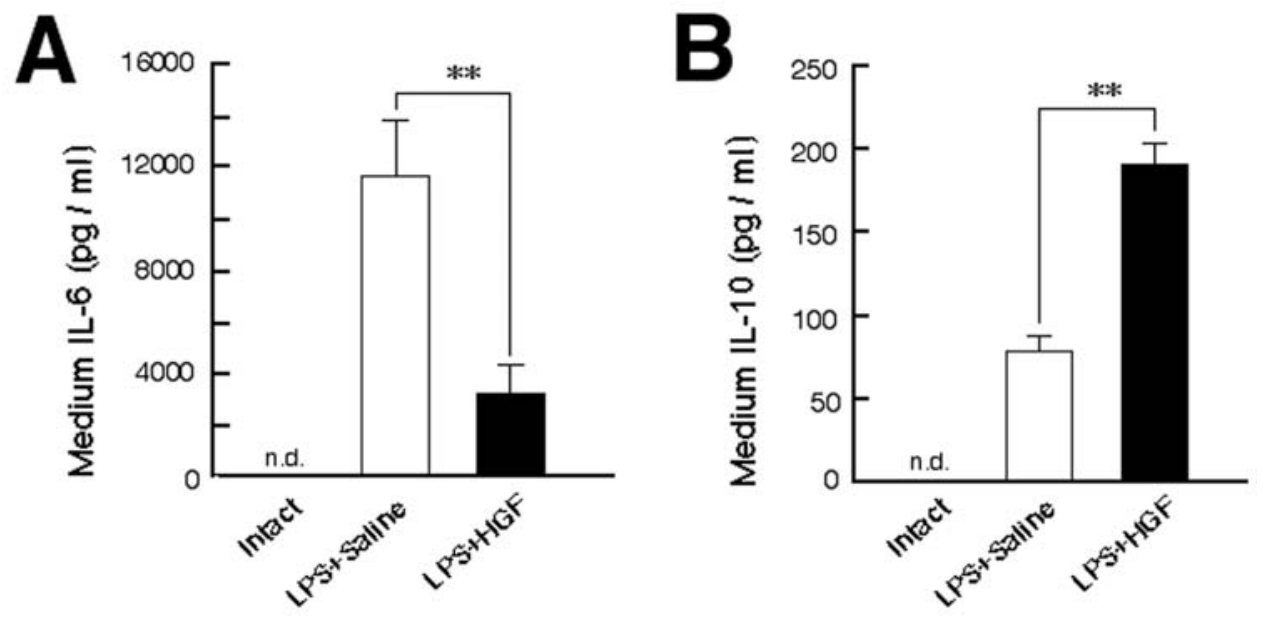

Figure 5. Changes in IL-6 and IL-10 levels by HGF in LPS-challenged macrophage in vitro. (A) Suppressive effect of HGF on LPS-mediated increase of IL-6 levels in the culture medium of Raw264.7 cells. (B) Enhancement of LPS-induced IL-10 production by HGF in this cell line. The culture supernatant was harvested $12 \mathrm{~h}$ after LS challenge and then subjected to ELISA. Data are expressed as mean $\pm \mathrm{SD},(\mathrm{n}=6)$. n.d., not detected. Statistical differences, ${ }^{* *} \mathrm{p}<0.01$.

LPS markedly increased IL-6 levels in the culture media of Raw264.7 cells. When LPS-treated Raw264.7 cells were coincubated with rh-HGF $(100 \mathrm{ng} / \mathrm{ml})$ for $12 \mathrm{~h}$, IL-6 levels declined to $25 \%$ of those in the LPS-treated cells (Fig. 5A), and this was associated with the concomitant increase in HO-1 levels (Fig. 4B). In contrast to the change in IL-6, HGF further enhanced the LPS-mediated increase of IL-10 levels in the culture medium of Raw264.7 cells (Fig. 5B). In the culture of mouse macrophages, SnPP repressed the HGF-induced increase in IL-10 levels by $>50 \%$, while the inhibition of IL- 6 

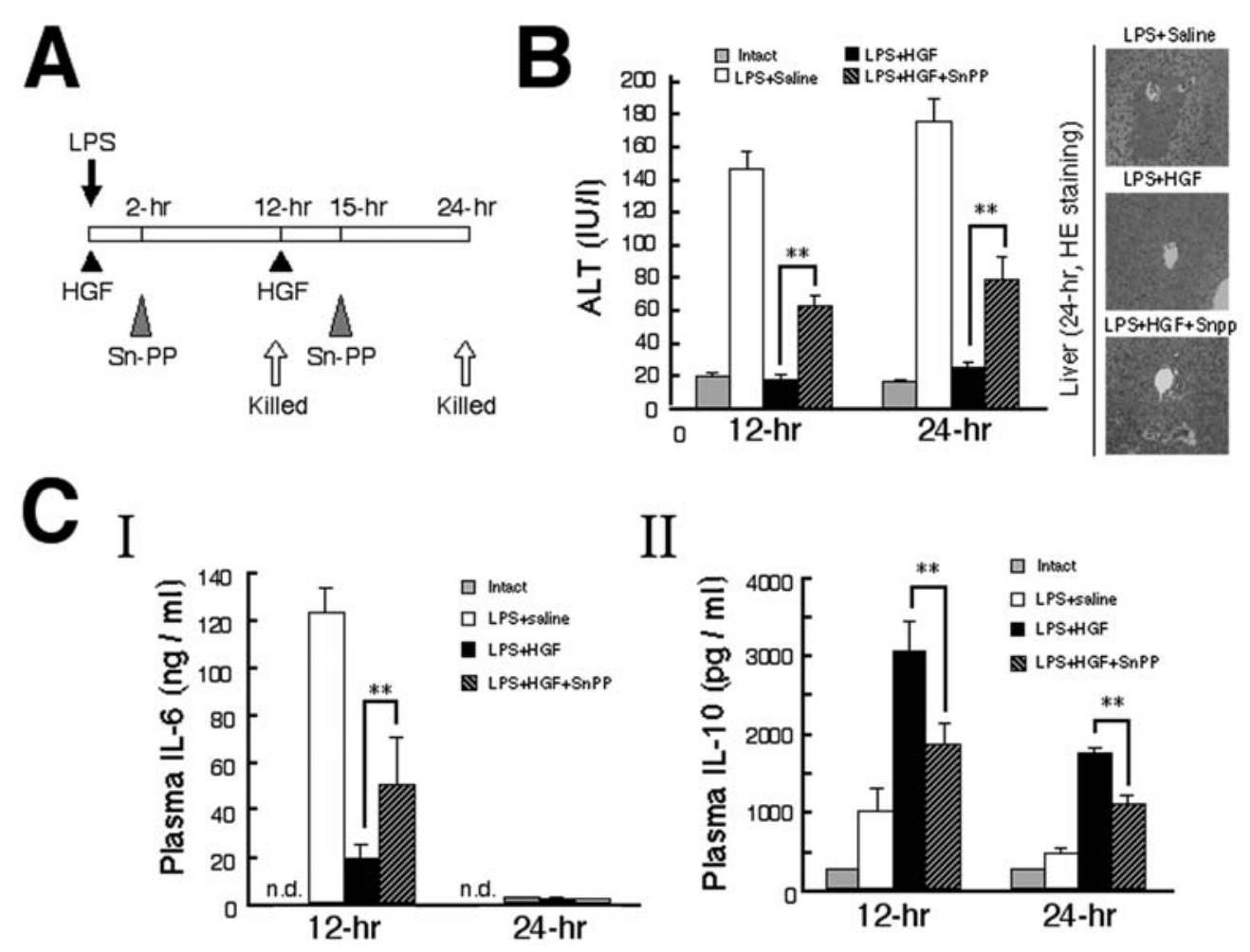

Figure 6. Effect of Sn-PP on HGF-mediated liver protection in LPS-treated mice. (A) Protocol for Sn-PP treatment during rh-HGF administration in LPStreated mice. (B) In vivo effects of Sn-PP on acute hepatitis. Left, changes in plasma AST levels in mice subjected to various treatments. Right, typical hepatic morphology, as observed in endotoxic mice treated either with saline, HGF, or HGF plus Sn-PP (HE staining, x180). (C) Alterations of IL-6 (I) and IL-10 (II) levels in plasma of endotoxic mice, treated with saline, HGF and HGF plus Sn-PP. Plasma was obtained from mice 12 and $24 \mathrm{~h}$ after the LPS challenge and then was subjected to ELISA. Data are expressed as mean $\pm \mathrm{SD},(\mathrm{n}=6)$. Statistical analysis, ${ }^{* *} \mathrm{p}<0.01$ compared with the saline group at the same time points.

production by HGF was in part restored by this HO-1 inhibitor (data not shown). These findings suggest that HGF-induced change in IL-6/IL-10 balance in vivo is, in part, due to a direct effect on macrophages.

Reversal of HGF-mediated protection by HO-1 inhibition in vivo. We returned to an animal model to determine if endogenous HO-1 contributes to the protective effects of HGF on hepatic injuries. For this purpose, Sn-PP was administered at $30 \mu \mathrm{M} / \mathrm{kg}$ (Fig. 6A), since this dose is widely used in numerous models of inflammation, with the apparent loss in HO-1 activity $(27,28)$. As shown in Fig. 1B, HGF inhibited the onset of liver injuries, as evaluated by plasma ALT levels. Of note, the protective effect of HGF against hepatic injury was partially reversed by Sn-PP injections. HGF treatment decreased plasma ALT levels in the LPS-treated mice to $12 \%$ of the saline control, while Sn-PP negated the HGF-mediated decrease in AST levels (Fig. 6B, left). Together with the increase in HO-1 levels (Fig. 3B), HGF reduced the area of necrotic and apoptotic tissue, as seen in the saline-treated mice up to $24 \mathrm{~h}$ after the LPS challenge. Of note, the combination of HGF plus Sn-PP reduced the HGF-mediated protective effects on hepatocytes (Fig. 6B, right). A significant difference was seen in the liver injury score between the HGF group and HGF plus Sn-PP group (24 h, 0.40 \pm 0.22 vs. $1.13 \pm 0.35, \mathrm{p}<0.05)$.

Finally, we evaluated the balance between IL-6/IL-10 to elucidate the mechanism whereby HGF-stimulated HO-1 results in therapeutic outcomes. As shown in Fig. 2, HGF increased the concentration of IL-10 in plasma and reduced IL-6 levels, between 12 and $24 \mathrm{~h}$ after the LPS challenge. Sn-PP partially reversed the HGF-induced reciprocal changes in IL-6 and IL-10 in septic mice (Fig. 6C). HGF reduced the plasma IL-6 levels to $16 \%$ of saline control $12 \mathrm{~h}$ after the LPS challenge. This effect was partially reversed by Sn-PP (Fig. 6C-I). As shown in Fig. 2, there was an apparent increase in plasma IL-10 levels $12 \mathrm{~h}$ post-LPS-challenge, while Sn-PP suppressed the HGF-mediated up-regulation of IL-10 by $>50 \%$ (Fig. 6C-II).

\section{Discussion}

There is now ample evidence that $\mathrm{HO}-1$ is required for an anti-inflammatory response during pathological conditions, including septic stresses $(6,7)$. In the present study, we examined the effect of HGF on macrophages for the following reasons, (i) LPS up-regulates c-Met expression in macrophage in vitro (20); (ii) HO-1 is induced in hepatic macrophages of humans with septic conditions $(7,26)$; and (iii) IL-6 and IL-10 are produced mainly by macrophages $(4,5)$, while $\mathrm{HO}-1$ is a key regulator of these cytokines (9). Using an animal model of endotoxemia, we found herein that HGF increases expression levels of HO-1 in hepatic macrophages, accompanied by protection of this organ. Of interest, an HO-1 inhibitor partially diminished the HGF-mediated protective effect against endotoxemia-induced injury. Based on the present results, we conclude that HGF-induced enhancement of HO-1 levels in macrophages is, at least in part, responsible for the host 
defense system under endotoxemia. This is the first report to show the role of HO-1 in HGF-produced protective outcomes under pathological conditions.

One key finding is that p-Met signals were mainly detected in Mac1-positive cells (and partially in hepatocytes) after rh-HGF treatment, thus indicating an importance of macrophages as an initial target of HGF. We reported that HGF inhibits the loss of hepatocytes in rats after LPS challenges (29), while it is known that the products of the HO-1-catalyzed reaction (such as carbon monoxide and biliverdin) protect hepatocytes from various injuries $(30,31)$. Thus, it is possible that during endotoxemia HGF protects hepatocytes via a release of HO-1-mediated products, such as carbon monoxide, from neighboring macrophages. In contrast, HGF blocked severe hepatitis in mice caused by Fas-agonist (jo2) (16), through a direct effect of HGF on hepatocytes (32). In this model, however, jo2 attacks hepatocytes, while HGF also acts on c-Met of hepatocytes, resulting in anti-apoptosis via sequestration of Fas by c-Met (32). In other words, HGF targets and activates c-Met tyrosine in 'cells undergoing initial pathological events' (i.e., LPS-challenged macrophages or Fas-stimulated hepatocytes). Such a selective signaling via $\mathrm{HGF} / \mathrm{c}-\mathrm{Met}$ in injured cells (e.g., macrophage $>$ hepatocytes, within $12 \mathrm{~h}$ after LPS treatment) may be reasonable for inhibiting 'up-stream' pathological events.

It is important to elucidate the mechanism by which HGF enhances HO-1 expression in mice. Using an in vitro model, we delineated a direct action of HGF toward LPS-treated macrophages. It is still difficult to explain the failure of HGF alone to induce HO-1, since the p-Met signal was different between LPS-untreated Raw264.7 cells and intact mice. One way or another, the fact that HGF alone does not induce HO-1 indicates that the enhancing effect by HGF is 'not additive'. Thus, our attention was focused on the role of HGF in 'LPSinduced' HO-1 expression. Based on the results shown in Fig. 4, we predict that HGF increases HO-1 levels in LPS-treated macrophages, via a post-transcriptional pathway, as reported (33). Little is known about the mechanism by which HO-1 is degraded during inflammation, but HGF may inhibit the cascade that leads to degradation of HO-1. Another possibility is that HGF directly induces HO-1 expression in interstitial cells, other than macrophages, as reported (34). Thus, we speculate that stabilization of HO-1 (in macrophages) as well as induction of HO-1 (in non-macrophages) produces the protective effect of HGF in vivo, and future studies are required to address this speculation.

How the HGF-mediated increase in HO-1 levels leads to a therapeutic outcome should be discussed. Under endotoxemic states, IL-10 is a key anti-inflammatory regulator (5). Indeed, there is a rapid progression of LPS-induced hepatitis in IL-10-knockout mice, as compared with wild-type mice (35). In our mouse model, HGF increased plasma IL-10 levels, while this effect was diminished by HO-1-inhibition, suggesting that $\mathrm{HO}-1$ is an IL-10-inducer. Consistent with our findings, it was reported that HO-1 increases IL-10 production levels in macrophages both in vitro (36) and in vivo (37). Furthermore, the anti-inflammatory effects of HO-1 were abolished during IL-10 deficiency (37). Thus, we predict that the signaling cascade, starting with induction of HO-1 by HGF and continuing with the stimulation of IL-10 by HO-1, is a key mechanism by which HGF inhibits inflammatory disorders. Indeed, recent studies demonstrated that HGF increases IL-10 levels in rodents, associated with improvements in inflammation (38-40). Furthermore, in vitro, HGF increases IL-10 production directly, especially during the conversion of macrophage to dendritic cells (41). Thus, both the direct and indirect effects of HGF on $\mathrm{IL}-10$ production lead to anti-inflammatory responses during endotoxic stresses.

In contrast to IL-10, there is an intrinsic mechanism by which the progression of SIRS is accelerated. For example, IL-6 plays a causative role in organ failures during endotoxemia $(2,4)$. Actually, anti-IL-6 antibodies attenuated endotoxin-mediated pathological conditions, including fibrin deposition (42). Importantly, HO-1 is a key mediator of the counter-regulatory response against IL-6. For example, after LPS challenge, plasma IL-6 levels in HO-1-deficient mice are $>100$-fold greater than levels in wild-type mice (9). This observation supports the distinguished role of HO- 1 in inhibition of IL-6 production. Using LPS-treated mice, we found that HGF reduced plasma IL-6 levels and increased HO-1 levels. Sn-PP partially reversed this inhibitory effect, indicating that the $\mathrm{HGF} / \mathrm{HO}-1$ pathway is, at least in part, involved in down-regulation of IL-6. Indeed, HGF reduces IL-6 levels in rodents with local inflammation (43), whereas HO-1 is needed for protection against systemic or localized inflammation (6-9). HGF prevents hepatitis and suppressed fibrin deposition in septic rats (44). Collectively, we speculate that HO-1 is a down-stream effecter of HGF that has anticoagulant effects during endotoxemia.

Although we emphasized the importance of macrophage events, we consider the possibility that HO-1-independent pathway is also involved in HGF-mediated liver protection, since Sn-PP reversed the preventive outcome of HGF, but its effect was not complete. During endotoxemia, inflammatory cytokines, such as interferon- $\gamma$, are over-expressed (9), and interferon- $\gamma$ induces apoptosis of hepatocytes (45), thus indicating a down-stream pathological event. Of interest, HGF counteracts interferon- $\gamma$-induced apoptosis (45). Furthermore, HGF inhibits both apoptosis and necrosis in primary culture of hepatocytes $(29,46)$, via HO-1-independent mechanisms (32). Such direct effects of HGF on hepatocytes also participate in HGF-mediated liver protection (i.e., downstream mechanism). Overall, possible protective events are summarized as follows, (i) anti-apoptosis by HO-1-catalyzed products (such as carbon monoxide) $(30,31)$; (ii) regulation of IL-6/IL-10 balance (9); and (iii) counteractive effect of HGF on cytokine-mediated apoptosis (45). Under diseased conditions, HGF has anti-coagulant and anti-apoptotic properties in endothelium $(44,47)$ and promotes regeneration of resident epithelial cells $(14,18)$. Such multiple functions of HGF lead to beneficial outcomes under endotoxic conditions $(29,44)$.

Finally, we wish to postulate a potential use of rh-HGF (i.e., activated HGF) for treatment of sepsis. Physiologically, HGF is synthesized and secreted as an inactive single-chain precursor (i.e., pro-HGF) and then activated to a heterodimeric form by proteolytic processing (12). It is known that plasma HGF levels increase in septic patients (19) and in our model (not shown), but endogenous HGF is inactivated, since p-Met signal was not evident in LPS-treated mice without 
rh-HGF treatment (Fig. 3A-I). One possible explanation is that plasminogen activator inhibitor-1 (PAI-1) levels markedly increase in mice after LPS challenge (48), while PAI-I is required for inhibiting cleavage of pro-HGF to active form HGF (25). Given that pro-HGF is an antagonist of c-Met (49), the possible loss in HGF-activation is involved in pathogenesis of septic injury, as reported in other organ diseases $(25,50)$. Thus, administration of rh-HGF is considered as a novel option for attenuation of septic diseases $(29,44)$. Molecular studies on HGF/HO-1 axis would shed more light on the understanding of the host defense system of SIRS and other inflammatory and immunological diseases.

\section{Acknowledgments}

This work was supported by grants from the Ministry of Education, Science, Technology, Sports and Culture of Japan (No. 20590398 and 18590369 to SM, No. 17014060 and 18390087 to T.N., and the 21 st Century COE program to T.N.). We are grateful to Dr J. McDonald (Claremore, OK) for language assistance.

\section{References}

1. Matthay MA: Severe sepsis: a new treatment with both anticoagulant and anti-inflammatory properties. N Engl J Med 344: 759-762, 2001.

2. Antoniades CG, Berry PA, Wendon JA and Vergani D: The importance of immune dysfunction in determining outcome in acute liver failure. J Hepatol 49: 845-861, 2008.

3. Rolando N, Wade J, Davalos M, Wendon J, Philpott-Howard J and Williams R: The systemic inflammatory response syndrome in acute liver failure. Hepatology 32: 734-739, 2000.

4. Van der Poll T and van Deventer SJ: Cytokines and anticytokines in the pathogenesis of sepsis. Infect Dis Clin North Am 13: 413-426, 1999.

5. Oberholzer A, Oberholzer C, Moldawer LL, Kumar A and Creery WD: The therapeutic potential of interleukin-10 in infection and inflammation. Arch Immunol Ther Exp 48: 529-538, 2000.

6. Ryter SW, Alam J and Choi AMK: Heme oxygenase-1/carbon monoxide: From basic science to therapeutic implications. Physiol Rev 86: 583-650, 2006.

7. Farombi EO and Surh YJ: Heme oxygenase-1 as a potential therapeutic target for hepatoprotection. J Biochem Mol Biol 39: 479-491, 2006.

8. Kawashima A, Oda Y, Yachie A, Koizumi S and Nakanishi I: Heme oxygenase-1 deficiency: The first autopsy case. Hum Pathol 33: 125-130, 2002.

9. Kapturczak MH, Wasserfall C, Brusko T, Campbell-Thompson M, Ellis TM, Atkinson MA and Agarwal A: Heme oxygenase-1 modulates early inflammatory responses: evidence from the heme oxygenase-1-deficient mouse. Am J Pathol 165: 1045-1053, 2004.

10. Nakamura T, Nawa $K$ and Ichihara A: Partial purification and characterization of hepatocyte growth factor from serum of hepatectomized rats. Biochem Biophys Res Commun 122: 1450-1459, 1984.

11. Nakamura T, Nishizawa T, Hagiya M, Seki T, Shimonishi M, Sugimura A, Tashiro K and Shimizu S: Molecular cloning and expression of human hepatocyte growth factor. Nature 342: 440-443, 1989.

12. Funakoshi H and Nakamura T: Hepatocyte growth factor: from diagnosis to clinical application. Clin Chem Acta 327: 1-23, 2003.

13. Birchmeier $C$ and Gherardi E: Developmental roles of HGF/SF and its receptor, the c-Met tyrosine kinase. Trends Cell Biol 8: 404-410, 1998.

14. Ishiki Y, Ohnishi H, Muto Y, Matsumoto K and Nakamura T: Direct evidence that hepatocyte growth factor is a hepatotrophic factor for liver regeneration and for potent anti-hepatitis action in vivo. Hepatology 16: 1227-1235, 1992.

15. Mizuno $S$ and Nakamura T: Hepatocyte growth factor: A regenerative drug for acute hepatitis and liver cirrhosis. Regener Med 2: 161-170, 2007.
16. Kosai K, Matsumoto K, Nagata S, Tsujimoto Y and Nakamura T: Abrogation of Fas-induced fulminant hepatic failure in mice by hepatocyte growth factor. Biochem Biophys Res Commun 244: 683-690, 1998

17. Huh CG, Factor VM, Sánchez A, Uchida K, Conner EA and Thorgeirsson SS: Hepatocyte growth factor/c-met signaling pathway is required for efficient liver regeneration and repair. Proc Natl Acad Sci USA 101: 4477-4482, 2004.

18. Li Z, Mizuno S and Nakamura T: Anti-necrotic and antiapoptotic effects of hepatocyte growth factor on cholestatic hepatitis in a mouse model of bile-obstructive diseases. Am J Physiol Gastrointest Liver Physiol 292: G639-G646, 2007.

19. Sekine K, Fujishima S and Aikawa N: Plasma hepatocyte growth factor is increased in early-phase sepsis. J Infect Chemother 10: 110-114, 2004.

20. Galimi F, Cottone E, Vigna E, Arena N, Boccaccio C, Giordano S, Naldini L and Comoglio PM: Hepatocyte growth factor is a regulator of monocyte-macrophage function. J Immunol 166: 1241-1247, 2001.

21. Muftuoglu MA, Aktekin A, Ozdemir NC and Saglam A: Liver injury in sepsis and abdominal compartment syndrome in rats. Surg Today 36: 519-524, 2006.

22. Hsu HY, Chu LC, Hua KF and Chao LK: Heme oxygenase-1 mediates the anti-inflammatory effect of Curcumin within LPSstimulated human monocytes. J Cell Physiol 215: 603-612, 2008.

23. Tajima H, Higuchi O, Mizuno K and Nakamura T: Tissue distribution of hepatocyte growth factor receptor and its exclusive down-regulation in a regenerating organ after injury. J Biochem 111: 401-406, 1992

24. Mizuno S and Nakamura T: Hepatocyte growth factor: Physiological and therapeutic ligand to attenuate diabetic nephropathy. In: Diabetic Kidney. Mogensen CE and Cortes P (eds). Humana Press, Totawa, NJ, pp225-253, 2006.

25. Hattori N, Mizuno S, Yoshida Y, Chin K, Mishima M, Sisson TH, Simon RH, Nakamura T and Miyake M: The plasminogen activation system reduces fibrosis in the lung by a hepatocyte growth factor-dependent mechanism. Am J Pathol 164: 1091-1098, 2004.

26. Clark IA, Awburn MM, Harper CG, Liomba NG and Molyneux ME: Induction of HO-1 in tissue macrophages and monocytes in fatal falciparum malaria and sepsis. Malar J 2: 41-53, 2003.

27. Reeve VE and Tyrrell RM: Heme oxygenase induction mediates the photoimmuno-protective activity of UVA radiation in the mouse. Proc Natl Acad Sci USA 96: 9317-9321, 1999.

28. Datla SR, Dusting GJ, Mori TA, Taylor CJ, Croft KD and Jiang F: Induction of heme oxygenase-1 in vivo suppresses NADPH oxidase derived oxidative stress. Hypertension 50: 636-642, 2007

29. Kosai K, Matsumoto K, Funakoshi H and Nakamura T: Hepatocyte growth factor prevents endotoxin-induced lethal hepatic failure in mice. Hepatology 30: 151-159, 1999.

30. Sass G, Seyfried S, Soares MP, Yamashita K, Kaczmarek E, Neuhuber WL and Tiegs G: Cooperative effect of biliverdin and carbon monoxide on survival of mice in immune-mediated liver injury. Hepatology 40: 1128-1135, 2004

31. Ke B, Buelow R, Shen XD, Melinek J, Amersi F, Gao F, Ritter T, Volk HD, Busuttil RW and Kupiec-Weglinski JW: Heme oxygenase 1 gene transfer prevents CD95/Fas ligand-mediated apoptosis and improves liver allograft survival via carbon monoxide signaling pathway. Hum Gene Ther 13: 1189-1199, 2002.

32. Wang X, DeFrances MC, Dai Y, Pediaditakis P, Johnson C, Bell A, Michalopoulos GK and Zarnegar R: A mechanism of cell survival: sequestration of Fas by the HGF receptor Met. Mol Cell 9: 411-421, 2002.

33. Lordnejad MR, Schliess F, Wettstein M and Häussinger D: Modulation of the heme oxygenase HO-1 expression by hyperosmolarity and betaine in primary rat hepatocytes. Arch Biochem Biophys 388: 285-292, 2001.

34. Radhakrishnan N, Bhaskaran M and Singhal PC: Hepatocyte growth factor modulates $\mathrm{H}_{2} \mathrm{O}_{2}$-induced mesangial cell apoptosis through induction of heme oxygenase-1. Nephron Physiol 101: 92-98, 2005

35. Zhong J, Deaciuc IV, Burikhanov R and de Villiers WJ: Lipopolysaccharide-induced liver apoptosis is increased in interleukin-10 knockout mice. Biochim Biophys Acta 1762: 468-477, 2006.

36. Chauveau C, Rémy S, Royer PJ, Hill M, Tanguy-Royer S, Hubert FX, Tesson L, Brion R, Beriou G, Gregoire M, Josien R, Cuturi MC and Anegon I: Heme oxygenase-1 expression inhibits dendritic cell maturation and proinflammatory function but conserves IL-10 expression. Blood 106: 1694-1702, 2005. 
37. Xia ZW, Xu LQ, Zhong WW, Wei JJ, Li NL, Shao J, Li YZ, Yu SC and Zhang ZL: Heme oxygenase-1 attenuates ovalbumininduced airway inflammation by up-regulation of foxp3 T-regulatory cells, interleukin-10, and membrane-bound transforming growth factor-beta1. Am J Pathol 171: 1904-1914, 2007.

38. Futamatsu H, Suzuki J, Mizuno S, Koga N, Adachi S, Kosuge H, Maejima Y, Hirao K, Nakamura T and Isobe M: Hepatocyte growth factor ameliorates the progression of experimental autoimmune myocarditis: a potential role for induction of $\mathrm{T}$ helper 2 cytokines. Circ Res 96: 823-830, 2005.

39. Okunishi K, Dohi M, Fujio K, Nakagome K, Tabata Y, Okasora T, Seki M, Shibuya M, Imamura M, Harada H, Tanaka R and Yamamoto K: Hepatocyte growth factor significantly suppresses collagen-induced arthritis in mice. J Immunol 179: 5504-5513, 2007.

40. Yamaura K, Ito K, Tsukioka K, Wada Y, Makiuchi A, Sakaguchi M, Akashima T, Fujimori M, Sawa Y, Morishita R, Matsumoto K, Nakamura T, Suzuki J, Amano J and Isobe M: Suppression of acute and chronic rejection by hepatocyte growth factor in a murine model of cardiac transplantation: induction of tolerance and prevention of cardiac allograft vasculopathy. Circulation 110: 1650-1657, 2004.

41. Rutella S, Bonanno G, Procoli A, Mariotti A, de Ritis DG, Curti A, Danese S, Pessina G, Pandolfi S, Natoni F, Di Febo A, Scambia G, Manfredini R, Salati S, Ferrari S, Pierelli L, Leone G and Lemoli RM: Hepatocyte growth factor favors monocyte differentiation into regulatory interleukin (IL)-10++IL-12 low/neg accessory cells with dendritic-cell features. Blood 108: 218-227, 2006.

42. Van der Poll T, Levi M, Hack CE, ten Cate H, van Deventer SJ, Eerenberg AJ, de Groot ER, Jansen J, Gallati H and Buller HR: Elimination of interleukin 6 attenuates coagulation activation in experimental endotoxemia in chimpanzees. J Exp Med 179: $1253-1259,1994$.

43. Azuma H, Takahara S, Matsumoto K, Ichimaru N, Wang JD, Moriyama T, Waaga AM, Kitamura M, Otsuki Y, Okuyama A, Katsuoka Y, Chandraker A, Sayegh MH and Nakamura T: Hepatocyte growth factor prevents the development of chronic allograft nephropathy in rats. J Am Soc Nephrol 12: 1280-1292, 2001.
44. Seto S, Kaido T, Yamaoka S, Yoshikawa A, Arii S, Nakamura T, Niwano M and Imamura M: Hepatocyte growth factor prevents lipopolysaccharide-induced hepatic sinusoidal endothelial cell injury and intrasinusoidal fibrin deposition in rats. J Surg Res 80: 194-199, 1998.

45. Morita M, Watanabe Y and Akaike T: Protective effect of hepatocyte growth factor on interferon- $\gamma$-induced cytotoxicity in mouse hepatocytes. Hepatology 21: 1585-1593, 1995.

46. Takehara $\mathrm{T}$ and Nakamura T: Protective effect of hepatocyte growth factor on in vitro hepatitis in primary cultured hepatocytes. Biomed Res 12: 335-338, 1991.

47. Mizuno S and Nakamura T: Prevention of neutrophil extravasation by hepatocyte growth factor leads to attenuations of tubular apoptosis and renal dysfunction in mouse ischemic kidneys. Am J Pathol 166: 1895-1905, 2005.

48. Yamamoto K, Shimokawa T, Yi H, Isobe K, Kojima T, Loskutoff DJ and Saito H: Aging accelerates endotoxin-induced thrombosis: increased responses of plasminogen activator inhibitor-1 and lipopolysaccharide signaling with aging. Am J Pathol 161: 1805-1814, 2002.

49. Mazzone M, Basilico C, Cavassa S, Pennacchietti S, Risio M, Naldini L, Comoglio PM and Michieli P: An uncleavable form of pro-scatter factor suppresses tumor growth and dissemination in mice. J Clin Invest 114: 1418-1432, 2004

50. Itoh H, Naganuma S, Takeda N, Miyata S, Uchinokura S, Fukushima T, Uchiyama S, Tanaka H, Nagaike K, Shimomura T, Miyazawa K, Yamada G, Kitamura N, Koono M and Kataoka H: Regeneration of injured intestinal mucosa is impaired in hepatocyte growth factor activator-deficient mice. Gastroenterology 127: 1423-1435, 2004. 